\title{
Labyrinthe
}

37 | 2011 (2)

Des séries et des vies

\section{Les Feux de la vie et de la beauté}

\section{Guillaume Paugam}

\section{(2) OpenEdition}

Journals

Édition électronique

URL : http://journals.openedition.org/labyrinthe/4185

DOI : $10.4000 /$ labyrinthe.4185

ISSN : 1950-6031

Éditeur

Hermann

\section{Édition imprimée}

Date de publication : 15 août 2011

Pagination : 23-34

ISBN : 9782705681470

\section{Référence électronique}

Guillaume Paugam, «Les Feux de la vie et de la beauté », Labyrinthe [En ligne], 37 | 2011 (2), mis en ligne le 01 août 2013, consulté le 20 avril 2019. URL : http://journals.openedition.org/labyrinthe/4185 ; DOI : $10.4000 /$ labyrinthe.4185 


\title{
Les Feux de la vie et de la beauté
}

\author{
Guillaume Paugam
}

À la une du Soap opera digest daté du 8 mars 2011, cinq photographies barrées d'un titre composé en capitales italiques, rouges et oranges, qui se détachent sur un fin halo blanc: "SHOCKING CAST SWITCHES! » ( CHANGEMENTS CHOQUANTS DE DISTRIBUTION! »).

Sur la rangée supérieure, à gauche du titre bleu cyan, l'image d'une femme, Natalia Livingston, jouxte celle d'une autre, Tamara Braun - reliées l'une à l'autre par le laconique verdict «Replaced! » («Remplacée! ») que surplombe la mention « Days » (pour Days of our lives, en français: Des jours et des vies). Le sens de la lecture recrée ainsi le jeu de chaises musicales qui les fait se succéder l'une à l'autre et que développe synoptiquement, de part et d'autre de cette mention, l'indication que la première est: « Out! », quand la seconde est: «In! » ${ }^{1}$. Ou, dit autrement, en miroir de ces images qu'elles légendent la ligne supérieure « Out ! / Days / In! » redouble l'inférieure « Natalie Livingston / Replaced! / Tamara Braun »; comme si seule cette triple répétition pouvait assurer de vaincre la réticence supposée du lectorat, sa prévisible stupéfaction, l'écho typographique de la disposition visuelle tente de figurer du choc le retentissement incrédule.

Occupant le tiers inférieur de cette même couverture, deux images encore, cette fois non légendées, où s'incruste, empiétant sur l'une et l'autre, cette information brute: "One life » (pour One life to live, en français: On ne vit qu'une fois), puis, sur deux lignes : « Todd / Swap! » («Changement de Todd!») - sans doute fallait-il, pour mieux mesurer l'énormité du bouleversement, bien détacher chacun des mots de cet invraisemblable coup de théâtre. Il s'agit de deux hommes, la trentaine avancée quoique fringuante, visage carré, regard d'acier - celui de gauche fixant droit l'œil inquisiteur du chaland. Entamé de part et d'autre par cet

1. En pages intérieures, la petite musique de Gainsbourg est remplacée par celle des Beatles; la même mise en scène photographique est commentée ainsi : "You Say Good-Bye, I Say Hello : Livingston (I.) was replaced by former GH co-star Braun » (Livingston a été replacée par Braun, l'ancienne héroïne d'Hôpital central). 
échange de vues, le jeu de séduction n'en est pas pour autant réciproque: l'acteur ayant figé son regard sur papier glacé s'est offert, sans retour immédiat, au lecteur qui en tient le sort littéralement entre ses mains.

Entre ces deux motifs enfin, occupant la première colonne mais disposée un peu en porte-à-faux, à peu près à la mi-hauteur de la page (de sorte qu'elle viendrait mordre sur les Todd si elle n'était, en fait, située sur un plan inférieur qui permet que son épaule gauche s'efface derrière le visage un peu interdit du partant), l'image d'une femme. L'air un peu hagard. Pensive. Déplacée. Ses yeux ne croisent pas l'objectif, par son regard elle semble déjà appartenir à un ailleurs - comme déjà absente: « GH's Brenda » (« Brenda de General Hospital », en français: Hôpital central); au-dessous et en plus gros caractères: «Gone! » («Partie! »). Entre ces images, les espaces sont comblés par des aplats jaune poussin et indigo. Tout en haut, un autre titre annonce, détachant sa lettrine blanche sur un bandeau de vert vif: « Ex-GH star turns down Days deal» («Une ex-vedette d'Hôpital central refuse un engagement dans On ne vit qu'une fois »). Entre passé et présent, sur le ton journalistique du constat ou familier de l'interpellation, la couverture a déjà embarqué le lecteur au cœur de son histoire.

Fondé en 1975, Soap opera digest revendique aujourd'hui 500000 abonnés et plus d'un million d'achats réguliers au numéro. L'hebdomadaire figure sur les présentoirs dédiés de nombreux supermarchés, au même titre que le plus récent Soap opera weekly (1989 - lancé à l'origine comme son complément avant d'être racheté par un groupe de presse concurrent, les deux magazines font à nouveau partie du même groupe de presse, Source Interlink Media, et font notamment site internet commun), et quelques autres moins prestigieux. Quoiqu'il enregistre un déclin relatif - dû probablement à l'affaiblissement d'audience des programmes couverts, à la concurrence agressive des magazines TV généralistes, et, bien entendu, au web - le magazine lui-même est presque une institution. Dans les années 1990, il a compté plus d'un million d'abonnés et c'est fort d'un tel succès quantitatif qu'il s'est érigé en instance légitimatrice de ces soap qu'il ne faisait jusqu'alors que chroniquer: du milieu des années quatrevingt et jusqu'en 2005 (à l'exception des années 1987, 2002 et 2004), il a décerné annuellement ses propres brevets d'excellence, les Soap opera digest awards. À peine plus réduit qu'un B5, son format est adapté au sac à main; on le retrouve en caisse, il s'achète $(\$ 3.99)$ en toute fin de courses et s'y glisse comme une gourmandise, un chapardage, un plaisir coupable. 
Le magazine a pour unique objet ces soap qu'il résume, dont il raconte les épisodes à venir et dévoile les coulisses; "soap » du nom des annonceurs privilégiés de ces programmes radio- puis télédiffusés à la mi-journée et qui visent la « ménagère-de-moins-de-cinquante-ans » : marchands de savon (soap) et/ou de lessive (soap powder), aux premiers rangs desquels « Lever Brothers », « Colgate-Palmolive » et la firme de Cincinnati « Procter \& Gamble ». «Opera » ? Adossée au soap, l'épithète ne semble y figurer que par dérision. C'est qu'on n'y chante guère (quoique l'on y fasse parfois chanter), les personnages ni la structure narrative ne correspondent à aucune œuvre effectivement composée, ils ne réfèrent aucunement à l'art lyrique et n'en miment jamais que la grandiloquence : violence des passions, des situations, des sentiments; retournement du sort, acharnement du destin, renversement dramatique; le soap rejoue à l'échelle de l'hyperbole et à horaire régulier cette extravagante comédie des rapports humains que l'implacable sobriété poétique des Québécois a traduit par: " roman-savon ». Récit et reflet, le magazine Soap opera digest a quelque chose de troublant, de fascinant, de presque invraisemblable tant il touche à la perfection fonctionnelle - accomplissant par sa forme même le motif de son contenu.

Égrainé le long de ses pages parsemées à leur tour d'annonces publicitaires dédiées au même public, le récit de ces programmes les redouble littérairement. C'est-à-dire qu'il en figure le portage graphique, non l'adaptation ou le complément. Loin de le supplanter, le magazine accompagne le soap. Partie intégrante de sa stratégie de séduction textuelle, la relation aguiche, attise et prolonge l'acte de visionnage : long préliminaire, la couverture journalistique se révèle encore un accessoire essentiel du jeu de dévoilement opéré par la diffusion qu'elle poursuit de surcroît au gré des analyses, commentaires de performances et autres confidences susurrées par ses acteurs en lisière de la scène. Bref, le magazine ne déflore l'action qu'en partie, ménageant les nervures dramatiques essentielles sur lesquelles il s'agira, la semaine prochaine, de revenir. Soap opera digest permet ainsi de feuilleter le «feuilleton »-du nom, selon le Littré, de l'article de critique artistique inséré en bas de page de journal qui restera ensuite à ces récits-fleuves qui ont fait les beaux jours de la presse du $\mathrm{XIX}^{\mathrm{e}}$ siècle (la périodicité de la rubrique laissant son nom au procédé de suivi de numéro en numéro d'une histoire toujours recommencée). En tant que redoublement textuel du soap, Soap opera digest ne fait donc que retourner à l'essentiel du feuilleton puisque, la redoublant, il 
en répète la structure verbale - cette longue phrase ininterrompue où l'événement, aussi terrible soit-il, ne figure guère qu'une péripétie; les intrigues parallèles autant de parenthèses; la catastrophe, rien de plus qu'une $n^{\text {ième }}$ inflexion.

Contrairement au film dont le schéma demeure largement aristotélicien (situation initiale, péripétie puis, à son climax, résolution - c'est-à-dire happy end), la grammaire du feuilleton se résume ainsi à sa syntaxe; son pivot est le point-virgule. L'arrêt y est en effet toujours provisoire; la fin d'une période marquant simultanément le début d'une nouvelle qui la relève, c'est-à-dire tout à la fois la remplace et la perpétue; ad libitum. La matière du soap sera donc la vie, ce feuilleton toujours recommencé auquel seule la mort peut donner un point final : puisque tout événement s'épuise, que son temps (chronos) se situe - et donc se déplace mais aussi se consume - le feuilleton n'aura d'autre histoire à conter que ce temps hors événement, le quotidien, dont l'intensité perpétuelle (aiôn) lui dure en arrière-plan. Ainsi le feuilleton français «L'Homme du Picardie » (ORTF, 1968) n'a-t-il gardé du film de Jean Vigo L'Atalante (1934) que la trame narrative: la vie quotidienne dans une péniche dont, justement, l'héroïne cinématographique avait cherché à fuir la monotonie. Le feuilleton prenait ainsi précisément pour objet cet instant figé (aussi statique et mouvant que son véhicule, la péniche), à l'exorcisation duquel se résumait toute l'existence de Juliette que la pellicule, pas plus que l'amour de son marinier de mari, ne pouvait fixer.

À l'inverse de la « série » (produit du croisement entre périodicité du feuilleton, récurrence de ses protagonistes et canevas narratif du film - c'est-à-dire de la tragédie ${ }^{2}$ ), le feuilleton ne propose pas de « saison » ni vraiment d' « épisode ». Il s'effeuille plutôt sur le principe d'un livre infini dont aucun chapitre ne consignerait sa trame propre, aucun volume annuel ne se proposerait l'exploration d'un thème particulier. Son principe est la superposition et non l'enchaînement; les événements de l'histoire ne s'accumulent pas, s'ils se succèdent c'est pour mieux se surimprimer - non pour se développer. La performance narrative du feuilleton est ainsi épuisée en une unique itération. Il ne se déploie ni ne se rejoue. D'abord parce que le feuilleton n'a pas de passé en dehors de ce présent, c'est-à-dire que son histoire a une valeur critique et non herméneutique: nulle vérité ne peut surgir de l'archive à rebours de ce qui est montré

2. En ce sens, les cycles sophocléens représentent sans doute le premier prototype de série. 
à présent, il n'est aucun indice caché, pas de matière à renversement, de rouage inattendu que la machine narrative aurait jadis déposé là et qu'il suffirait de remettre en branle pour faire surgir l'inoxydable vérité disposée en deçà de la corruption actuelle des apparences; le passé est là mais ni trace, ni témoignage, à peine pesanteur ${ }^{3}$. Si le feuilleton voit surgir d'un avant quelconque une information « nouvelle » (un membre de la famille retrouvé, un objet que l'on croyait perdu), ce sera pour confirmer son schéma tel qu'il est disposé maintenant, non pour amorcer sa réinterprétation intégrale qui invaliderait tout ce que le spectateur avait cru jusque-là. Les reconstitutions généalogiques (révélations d'origine, réattributions de paternité), les réduplications qui troublent l'identité (thème du double, du sosie, du jumeau), la transfiguration de la mort (ce décès auquel on avait faussement cru) finissent, au forceps, par s'intégrer à l'ordre rationnel du présent; ils ne viennent pas trahir le sol de la réalité sur laquelle ils s'appuient. Force du soap qui peut déroger à la vraisemblance sans sombrer dans le fantastique. L'acuité du moment présent est ainsi sans cesse réaffirmée, refermant toute possibilité de dénouement sur son cercle: ce qui est montré est le vrai, c'est-à-dire l'unique; ce qui est qui en a toujours été ainsi. Aussi, puisqu'il est toujours déjà-là, n'est-il commercialisé aucun enregistrement du feuilleton pour embrasser l'histoire, le spectateur n'a pour seuls recours que son assiduité, sa perspicacité à déduire du présent qui en découle ce passif qu'il évide et éventuellement sa capacité à faire le deuil de tel syntagme dont, justement, les magazines qui l'accompagnent tentent de consoler la perte. C'est à ce prix que le feuilleton s'écoule, inexorable car homogène au temps lui-même, et si les soap s'enorgueillissent de leur pérennité, c'est qu'elle leur permet de conjurer le fait que le passé leur apparaît comme une notion abstraite, fantomatique. La longévité permet alors d'homologuer ce temps dont ils n'ont pas la mémoire, lui substituant l'évidence factuelle d'une présence continue (des trois feuilletons cités et, à ce jour, encore en cours de production, Hôpital central est le plus

\footnotetext{
3. À l'opposé la série Monk (2002-2009) mettait en scène les enquêtes d'un détective talentueux tourmenté par la non-résolution d'un crime primitif, celui de sa propre épouse, sur lequel chaque saison apportait rétrospectivement de nouveaux éléments d'information. Le passé de la série figurait donc archétypiquement dans son présent au nom de sa valeur herméneutique : à son origine et motivant sa perpétuation jusqu'à une conclusion en forme d'apothéose, l'énigme se présentait ainsi doublement comme consubstantielle à la série - chaque enquête renvoyant pour sa part à la méta-enquête qui en formait la matrice de soubassement et dont l'élucidation marqua la fin du programme lui-même.
} 
ancien puisque sa production a démarré en 1963, Des jours et des vies date de 1965 et On ne vit qu'une fois de 1968).

Est-ce à dire que la représentation du quotidien, ou de son ennui, constitue le privilège du feuilleton sur la série? Voilà qui est deux fois faux. De nombreuses séries ont ainsi la vie quotidienne pour objet, le réel pour mise en scène, le contemporain en guise de théâtre. Mais leur grammaire est différente en son principe: quand le feuilleton est une expression de la vie, la série en est une reformulation; le feuilleton transcrit la vie, la série la transpose; il la prend comme sujet d'énonciation, elle en fait l'objet d'un jeu de langage - ce que l'on saisira mieux via un apologue de Hilary Putnam:

Pour les besoins des exemples de science-fiction qui vont suivre, nous allons supposer qu'il y a, quelque part dans la galaxie, une planète que nous appellerons Terre-Jumelle. Terre-Jumelle ressemble beaucoup à la Terre; en fait, les gens sur Terre-Jumelle parlent même français. À vrai dire, à l'exception des différences que nous préciserons dans nos exemples de science-fiction, le lecteur pourra supposer que TerreJumelle est exactement identique à notre Terre. [...] Une de ces particularités de Terre-Jumelle se trouve être que le liquide appelé « eau » n'est pas $\mathrm{H}_{2} \mathrm{O}$, mais un liquide différent dont la formule chimique est très longue et très compliquée. J'abrégerai cette formule chimique par XYZ. Je supposerai que XYZ est indiscernable de l'eau aux températures et aux pressions normales. En particulier, ce liquide a le même goût que l'eau, et, comme l'eau, il étanche la soif. Je supposerai également que les océans, les lacs et les mers de Terre-Jumelle contiennent de l'XYZ et non de l'eau, qu'il pleut sur Terre-Jumelle de l'XYZ et non de l'eau, etc. $\mathrm{Si}$, un jour, un vaisseau spatial en provenance de la Terre visite Terre-Jumelle, on supposera d'abord que « eau » a la même signification sur Terre et sur Terre-Jumelle. Cette hypothèse sera corrigée lorsqu'on aura découvert que l'« eau » sur Terre-Jumelle est de l'XYZ et le vaisseau spatial terrien fera à peu près le rapport suivant: "Sur Terre-Jumelle, le mot "eau" signifie XYZ. » ${ }^{4}$

4. H. Putnam, «Les significations ne sont tout simplement pas dans la tête» in P. Ludwig [éd.], Le Langage, (« The meaning of "meaning" », 1975), Paris, Flammarion « corpus », 1997. 
Comme cette Terre-Jumelle de la fable de Putnam, la série ne montre pas le quotidien, elle le diffracte au moyen d'une incongruité fondamentale qui déclenche toutes les autres. Dans Who's the boss? (1984-1992, en français: Madame est servie), le ressort est la métathèse - c'est-à-dire une permutation: une rigide femme d'affaire du Connecticut engage un Italo-Américain au cœur tendre en guise d'homme à tout faire. Dans Home improvement (1991-1999, en français: Papa bricole), il s'agit de l'hypallage - l'anomalie d'attribution consistant dans le fait que Tim, présentateur à succès d'une émission télévisée de bricolage, est incapable d'user correctement d'un tournevis; aussi son désir de mettre en application chez lui l'enseignement du programme dont il a la charge provoque-t-il immanquablement des catastrophes. Love \& Marriage (1987-1997, en français: Mariés, deux enfants) se déploie comme une tapinose: un père qui déteste son travail, une mère nymphomane, des enfants écervelés et obsédés, les Bundy forment cette hyperbole inversée qui représente en négatif la parfaite famille américaine. Le Cosby show (1984-1992) pour sa part se lit comme une diaphore où un même mot est entendu dans un sens différent selon son contexte puisque tout s'y déroule conformément au désordre d'une famille de la classe moyenne typique, à ceci près qu'elle est afro-américaine. La description proposée par Roseanne (1988-1997) paraît enfin caractéristique d'une hypotypose : dans une petite ville de l'Amérique moyenne, une famille en surpoids et aux faibles revenus aborde les questions relatives à l'alcool, la drogue, au racisme ou encore à l'homosexualité, etc. Chacune de ces séries - et tant d'autres - représente certes la vie, mais telle qu'elle aurait lieu sur une « Terre-Jumelle » qui diffère de notre monde en un point essentiel autour duquel se construit l'interprétation d'ensemble. Le monde est offert selon l'ordre rassurant d'un langage ordinaire mais contrefait, faisant vaciller le sens des mots au moment où scintille de son plus vif éclat le sol rassurant de la grammaire. À l'abri de toute contagion, le spectateur est ce voyageur de la fable de Putnam explorant comme un nouvel univers, mais obscurément familier, redécouvrant son propre monde au miroir de cette similarité quasi parfaite qu'il perçoit du commode vaisseau spatial que constitue son téléviseur. Il a ainsi sous les yeux une structuration d'ensemble qui n'est qu'infimement affectée par ce déplacement - tout est là, certes, mais curieusement disposé et donc étrangement inquiétant. De sorte que l'image, que les dialogues, que la phrase de la série même, tout y est saturé par le sous-texte des significations à peine altérées qui 
minent l'intrigue et dont seul le spectateur a la clef. La scène devient insensiblement matière à quiproquo existentiel (du fait de la différance entre Terre-Jumelle de la série et monde connu du spectateur), déclenchant cette hilarité qui va ponctuer l'histoire mais aussi lui donner sa raison - et donc son liant. Car la série a gardé de la Poétique la nécessité d'une unité d'action (l'excroissance est malvenue qui sera éventuellement traitée ailleurs, en une série dérivée ou spin-off) quand le feuilleton doit maintenir à tout moment une multiplicité d'intrigues parallèles. Alors que le soap se vit comme un drame continué, la série dont l'objet est le quotidien a ainsi pour vocation d'être drôle: le rire constitue son terminus a quo et ad quem; elle puise dans le jeu de langage sur lequel elle est construite le ressort comique qui la verra s'éteindre quand il sera tari ${ }^{5}$.

Le feuilleton pour sa part montre la vie telle qu'elle se déroule - non en l'imitant, mais en en capturant l'essence. C'est-à-dire qu'il n'a pas besoin de la refléter pour la réfléchir. De là le lot d'imprécisions qui va concourir à sa justesse: le théâtre de ses péripéties est certes situé, mais non localisable; ses personnages sont définis socio-professionnellement mais l'inutile détail de leur activité nous est épargné; l'omniprésente question de l'union est soigneusement dissociée du spectacle des ébats; bref, le soap offre toutes les garanties de l'identité sans le fardeau de l'identification. De cet ailleurs où il se déroule je connais peu l'horizon dessiné à grands traits dont je n'ai nul besoin - ce que je vois là, cette vie n'est pas la mienne, et c'est précisément ce qui fait son attrait. Tout dans cette vie n'est pas parfait, mais tout y est exemplaire. Temps, lieu et action n'ont de sens que comme cadre et non comme facteurs d'une prétention obsolète à la mimesis; la poétique du feuilleton n'a cure de veiller à leur unité non plus qu'à leur réalisme: ils ne valent guère qu'en tant qu'éléments vagues d'une mise en situation, l'intérêt de leur mise en scène sombre où commencent les privilèges de l'ekphrasis.

Si le feuilleton n'a pas à convaincre de son authenticité pour être vrai, c'est que son irréalité de principe permet au contraire de mieux amorcer l'effectivité des émotions qu'il met en jeu: mensonge, trahison et amour selon une palette qui va de l'attrait à la possession en passant par la haine, la passion, la folie; vengeance, attachement et culpabilité, etc. Le feuilleton

5. On distinguera cependant de ce quotidien montré absolument sa déclinaison génitive moins nécessairement comique - on entend par là les quotidiens $d e . .$. (policiers, avocats, médecins ou encore mafiosi comme c'est le cas pour Les Sopranos, etc.). 
substitue au spectre de la vulgarité du réel l'univers éthéré d'une hétérotopie quotidienne où, débarrassés de la chape du vraisemblable, les sentiments sont librement exaltés. La péripétie n'a ainsi de valeur qu'en tant qu'elle permet de prolonger l'intensité de la situation exposée, réactivant son passé, présageant son futur. C'est sur le fond de cette tension atemporelle que l'événement surgit - sa construction importe peu, son origine peut demeurer obscure, sa crédibilité n'est pas engagée. Il n'a de valeur que dans son être-là; il se présente, et son apparition va renouveler cette situation sur laquelle il prend sol, il apparaît et s'efface tôt comme prétexte tant il est vrai que le besoin d'explication s'éteint sous le désir de commentaire. Il n'est donc pas d'ennui dans le feuilleton, au sens où il n'est de variation si infime dont la minutieuse description ne dessine le motif de nouveaux développements, où il n'est aucun tropisme que la description adéquate ne puisse élever aux proportions de l'épopée.

L'on comprend alors comment le soap, en tant qu' absolu du feuilleton, a préparé l'avènement d'une « télé-réalité » (programme pas moins scénarisé que lui, dont le casting ne sera pas moins travaillé et dont l'intense langueur ponctuée juste à temps d'épisodes destinés à l'entretenir ne sera pas moins savamment ménagée) qui n'est pas parvenue à le supplanter. Elle avait beau lui emprunter en plus cru toute sa grammaire - sa syntaxe (la fixité d'une situation comme une longue phrase entrecoupée de plans serrés sur l'émotion), la gamme de ses réactions méthodiquement conjuguées (perplexité, mise en intrigue, accablement), les déclinaisons offertes par ses multiples ressorts dramatiques (unions et ruptures dont l'absoluité se verra surmontée à force d'amitié, de résilience, de volonté) - elle y manquait en un point capital: le soap ne transforme pas la vie mais il la remplace. Aussi avait-elle beau être plus authentique, il demeurait plus vrai. La preuve étant qu'aucune télé-réalité ne peut indéfiniment maintenir captifs les éléments qu'elle mobilise, tandis que le feuilleton tient en permanence son réel sous cloche - ainsi ces remplacements de casting chroniqués avec force exclamations par le magazine: dans l'univers immuable de ces existences fictives, une svelte trentenaire blonde, cheveux mi-longs savamment ondulés aux yeux marron remplace une svelte trentenaire blonde, cheveux mi-longs savamment ondulés aux yeux marron; un « Todd » blond aux yeux bleus, bouc taillé de près, mâchoire carrée, t-shirt sombre, s'apprête à remplacer un « Todd» blond aux yeux bleus, mal rasé, mâchoire carrée, chemise anthracite. Et quand « Brenda » fera ses adieux, le scénario ne manquera pas de ménager et sa disparition et sa réapparition possible. 
À l'inverse de la série qui en propose un miroir, le feuilleton forme ainsi de la vie une sorte de précipité: restitué selon un protocole expérimental, le condensé pur de ses émotions les mène invariablement à leur paroxysme (l'attachement au mariage, le dédain à l'homicide, le désordre au cataclysme) en vertu de leur extrême lisibilité ; l'existence y est déposée comme à livre ouvert. Aussi, quand l'expérience de la série est en effet philosophique, celle du feuilleton est-elle d'emblée littéraire: le feuilleton se déroule selon cette longue phrase continue, commencée inopinément par une majuscule; achevée, un jour peut-être, par un point; en l'attente, rythmée et tenue en suspens par ses innombrables points-virgules. Il va chroniquer la vie et non la mettre en abyme. D'où la parfaite circularité du retournement accompli par sa déclinaison magazine par lequel la vie des héros de feuilletons eux-mêmes finit par être couchée sur papier, racontée façon soap: narré en page intérieure, le parcours de Kristolyn Lloyd, de son Texas natal à l'Indonésie pour finir sur le plateau d'On ne vit qu'une fois; l'enquête sur les secrets des vedettes de soap les plus sexy qui occupe des pages 73 à 85 ; le quotidien de l'animal de compagnie de Chrishell Stause (Amanda, dans «AMC » pour All my children - en français: La Force du destin [depuis 1970]); l'édifiant bilan de ce que, vingt ans après leur première apparition, les acteurs Michael Easton, John J. York, Heather Tom et quelques autres, sont devenus (le premier est passé des Jours de notre vie à On ne vit qu'une fois, les deux autres sévissent toujours, respectivement, à Hôpital central et The Young and the restless [en français: Les Feux de l'amour; depuis 1973] qui ont vu leurs débuts - spectacle d'une rassurante immuabilité, tant et si bien que pour s'assurer du contraste, il a fallu passer les photos anciennes au filtre du noir \& blanc); ce clin d'œil remarquable dans The Bold and the Beautiful (en français : Amour, gloire et beauté, depuis 1987) de Bill à Olivia (qui, le temps d'un cross-over est passé des Feux de l'amour à Amour, gloire et beauté, rencontrant un personnage qu'incarne Don Diamont, soit le même qui jouait Brad, ex-amant d'Olivia, quand il sévissait dans Les Feux de l'amour): «Hum... Donc vous êtes certaine que nous ne nous sommes jamais rencontrés? D'une certaine manière, j'ai l'impression de vous connaître ${ }^{6}$. » Soap opera digest ne mime pas le soap comme le ferait un vulgaire roman-photo, il parachève plutôt son édifice structural : demeurait en effet seule obscure cette autre émotion qui échappe au

6. «So you're sure we haven't met, huh? You somehow look familiar to me. » 
soap, motivant la réalité-télé (et, pour peu que son surgissement soit mal maîtrisé, peut la faire voler en éclats) - celle de ses acteurs.

Aussi, peu dupes de l'illusion théâtrale qui, sous prétexte de confusion parvient toujours à désintriquer le sort de ses héros ("à la scène ») du destin de ceux qui les incarnent (« à la ville »), les magazines entreprennent de redoubler le récit du soap en cette bordure extrême de ce qu'il ne peut montrer avec la relation de l'existence de ceux qui le personnifient. Soap opera digest s'empare ainsi de la grammaire du soap qui rejoue la vie pour mieux encore l'assimiler à la vie - à commencer par la structure, qui unit la fiction, les mémoires et le commentaire, jusqu'à la syntaxe avec ses phrases sans rivages (ce Nil du langage qui déborde ici, pour les fertiliser, sur les plaines de la vérité). Les héros de ces soap auquel, comble de la familiarité, il a donné des surnoms deviennent ainsi le sujet de l'hyper-soap qui le prolonge et le nourrit; qui l'irrigue, témoins de ses bonheurs et de ses drames - la romance entre Bill Hayes et Susan Seaforth, acteurs des Jours et des vies, qui aboutira à leur mariage « réel » en 1974 avant celui, plus tumultueux, de la fiction (à deux reprises suivies chacune d'un divorce puis d'une réconciliation); le décès d'Helen Wagner qui aura tenu le rôle de Nancy Hughes dans As the world turns (inédit en France) de son premier épisode en 1956 à mai 2010, s'éteignant à peine deux mois avant la fin de la production du soap qu'elle avait vu naître et qui l'a enterrée (décision actée au préalable alors qu'elle était déjà malade - cancer et baisse d'audience, l'actrice nonagénaire et le feuilleton hexadécennal se sont mutuellement condamnés, comme si l'un à l'autre ne pouvait se survivre) ${ }^{7}$. Dans cette narration redoublée, mêlée et toujours recommencée le soap se confond ainsi avec son récit, le récit avec la vie - s'accroissant jusqu'à pouvoir durer aussi longtemps que la vie, jusqu'à lui équivaloir, pour détourner la célèbre phrase de la Recherche du temps perdu. Ne s'agit-il pas, d'ailleurs, du même procès? Dans cette capacité à annihiler le temps de l'événement dans celui de son attente, de son ressenti, de son ressouvenir qui le déjoue; dans la mise en scène de cette idée rêvée de la vie où se surimprime celle, transfigurée, de ses acteurs et à laquelle ses auteurs sacrifient intrigue, unité de la personne, cours du récit, jeu de l'imagination, ne faut-il pas reconnaître

7. L'acteur Charlie Sheen au contraire s'est vu évincé de la série Two and a half men (en français: « Mon oncle Charlie ", depuis 2003) dont il incarnait le personnage principal pourtant largement inspiré de son propre caractère: sa vie, devenant feuilleton, la reléguait à l'arrière-plan. 
l'effet propre du brouillage auto/bio/graphique jadis opéré par Proust? Les jours de Balbec préfigurent sans doute en quelque part ceux de Salem (qui sont ceux de nos vies); Côte ouest rejoue à sa manière le Côté de Guermantes - l'intensité du présent culminant çà et là dans ce temps en permanence perdu et retrouvé, dans la violence de l'attente soulagée par la délivrance du commentaire, dans la transfiguration imparfaite des signes de l'événement qui finissent par se substituer à lui, mystifié au creux d'un temps aboli, resserré, replié sur lui-même. En perpétuant le soap qu'il redouble, par cette tension du récit que porte la ponctuation, surchargée par celle du plan serré sur les visages inertes de ses acteurs, insensiblement traversés par cette émotion indicible qui les étreint, Soap opera digest ne fait donc que réactiver les privilèges narratif et syntaxique de ce labyrinthe textuel en lequel il s'origine, réaffirmant la primauté de la recréation sur la représentation - soit l'empire de la littérature sur la vie via l'état critique de son langage.

\section{Sources:}

- Un segment de phrase emprunté à Laurent Dubreuil, L'État critique de la littérature, Paris, Hermann, 2009 (sur La Recherche du temps perdu).

- Trois citations de W. Benjamin, «L'Image proustienne » in Euvres $I I$ [trad. par M. de Gandillac revue par R. Rochlitz], Paris, Gallimard « folio », 2000 [1929].

- www.wikipedia.org

PS : Alors que nous mettions sous presse, nous avons appris que le réseau $A B C$ qui a déjà annoncé cesser la diffusion de La Force du destin et On ne vit qu'une fois pourrait également renoncer à Hôpital centrals. Alors, ces rumeurs sont-elles fondées? Le réseau CBS reprendra-t-il les diffusions abandonnées par $A B C$ ? Cette inflexion dans la programmation d'ABC marque-t-elle un tournant irrémédiable dans l'histoire du soap - son lent déclin, son nécessaire renouveau?

8. « General Hospital cancellation rumors grow » [URL $=$ http://soapcentral.com/gh/news/2011/0505cancellation.php, consultée le 23/05/2011]. 